\title{
openheart Subclinical cardiopulmonary dysfunction in stage 3 chronic kidney disease
}

\author{
Alexander Nelson, ${ }^{1}$ James Otto, ${ }^{2}$ John Whittle, ${ }^{3}$ Robert C M Stephens, ${ }^{4}$ \\ Daniel S Martin, ${ }^{2}$ John R Prowle, ${ }^{5}$ Gareth L Ackland ${ }^{5}$
}

To cite: Nelson A, Otto J, Whittle J, et al. Subclinical cardiopulmonary dysfunction in stage 3 chronic

kidney disease. Open Heart 2016;3: $\mathrm{e} 000370$.

doi:10.1136/openhrt-2015000370

- Additional material is available. To view please visit the journal (http://dx.doi.org/ 10.1136/openhrt-2015000370).

Received 24 November 2015 Revised 9 January 2016 Accepted 12 January 2016

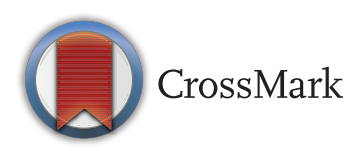

${ }^{1}$ UCL Medical School, London, UK

${ }^{2}$ Royal Free London NHS Foundation Trust, London, UK

${ }^{3}$ Division of Medicine, Department of Clinical Physiology, University College London, London, UK ${ }^{4}$ Department of Anaesthesia, University College London Hospitals NHS Trust, London, UK

${ }^{5}$ William Harvey Research Institute, Queen Mary University of London, London, UK

Correspondence to Dr Gareth L Ackland; g.ackland@qmul.ac.uk

\section{ABSTRACT}

Objective: Reduced exercise capacity is well documented in end-stage chronic kidney disease (CKD), preceded by changes in cardiac morphology in CKD stage 3. However, it is unknown whether subclinical cardiopulmonary dysfunction occurs in CKD stage 3 independently of heart failure.

Methods: Prospective observational cross-sectional study of exercise capacity assessed by cardiopulmonary exercise testing in 993 preoperative patients. Primary outcome was peak oxygen consumption $\left(\mathrm{VO}_{2 \text { peak }}\right)$. Anaerobic threshold (AT), oxygen pulse and exercise-evoked measures of autonomic function were analysed, controlling for CKD stage 3, age, gender, diabetes mellitus and hypertension.

Results: CKD stage 3 was present in 93/993 (9.97\%) patients. Diabetes mellitus (RR $2.49(95 \% \mathrm{Cl} 1.59$ to $3.89) ; p<0.001)$, and hypertension (RR $3.20(95 \% \mathrm{Cl}$ 2.04 to 5.03); $p<0.001)$ ) were more common in CKD stage 3. Cardiac failure (RR $0.83(95 \% \mathrm{Cl} 0.30$ to 2.24); $p=0.70$ ) and ischaemic heart disease (RR 1.40 (95\% Cl 0.97 to 2.02); $p=0.09$ ) were not more common in CKD stage 3. Patients with CKD stage 3 had lower predicted $\mathrm{VO}_{2 \text { peak }}$ (mean difference: $6 \%$ (95\% Cl 1\% to 11\%); $p=0.02)$, lower peak heart rate (mean difference:9 bpm (95\% Cl 3 to 14); $p=0.03)$ ), lower AT (mean difference: $1.1 \mathrm{~mL} / \mathrm{min} / \mathrm{kg}(95 \% \mathrm{Cl} 0.4$ to 1.7); $p<0.001$ ) and impaired heart rate recovery (mean difference: $4 \mathrm{bpm}(95 \% \mathrm{Cl} 1$ to 7$) ; \mathrm{p}<0.001)$ ). Conclusions: Subclinical cardiopulmonary dysfunction in CKD stage 3 is common. This study suggests that maladaptive cardiovascular/autonomic dysfunction may be established in CKD stage 3, preceding pathophysiology reported in end-stage CKD.

\section{INTRODUCTION}

Chronic kidney disease (CKD) is associated with increased cardiovascular risk ${ }^{1}$ and mortality $^{2}$ and increased incidence of cardiac failure. ${ }^{3-5}$ Reduced exercise capacity in patients with end-stage renal disease ${ }^{6}$ is associated with poor survival. $^{7-9}$ In patients with end-stage renal disease, the combination of increased left ventricular mass, myocardial fibrosis, elevated

\section{KEY QUESTIONS}

What is already known about this subject?

- Early chronic kidney disease (CKD) is associated with structural cardiac changes, but the functional significance of this has not been explored.

\section{What does this study add?}

- Using cardiopulmonary exercise testing, we show that early CKD is associated with subclinical, inferior cardiopulmonary reserve and evidence for autonomic dysfunction.

How might this impact on clinical practice?

- These data contribute new insights into why early CKD appears to be associated with worse clinical outcomes across a range of clinical scenarios, including percutaneous coronary intervention, cardiac and non-cardiac surgery.

left ventricular filling pressure and arterial stiffness are associated with reduced exercise capacity. ${ }^{10}$ Similarly, autonomic abnormalities have been described in end-stage (haemodialysisdependent) CKD. ${ }^{11}{ }^{12}$ By contrast, there is surprisingly few data on the association between impaired cardiac and/or autonomic function in asymptomatic patients with early CKD (CKD stage 3). Although changes in cardiac structure occur at different stages of estimated glomerular filtration rate (eGFR), ${ }^{13}$ no significant associations between kidney function and systolic or diastolic cardiac function have been found even after adjusting for potential confounding variables. $^{13}$ In this prospective, cross-sectional observational study, we hypothesised that subclinical cardiopulmonary dysfunction in CKD stage 3 may be unmasked by objectively assessing cardiopulmonary reserve using cardiopulmonary exercise testing (CPET).

\section{METHODS}

IRB approval (MREC: 12/LO/0453) was obtained in accordance with the Declaration of Helsinki. Adherence to STROBE 
guidelines is documented in online supplementary material. Patients were enrolled prospectively at University College London Hospitals, UK (from December 2007 to May 2014), referred by their clinical team for CPET as part of routine standard-of-care preoperative assessment. Patients gave written consent to use CPET data. Exclusion criteria for CPET were in accordance with international guidelines. ${ }^{14}$ Adherence to STROBE guidelines is documented in the online supplementary material.

\section{CKD assessment}

eGFR was calculated from routine preoperative creatinine measurements using the modification of diet in renal disease (MDRD) equation, ${ }^{15}$ in line with most previous perioperative studies in the non-cardiac surgical populations. As we were primarily interested in categorising eGFR $<60 \mathrm{~mL} / \mathrm{min} / 1.73 \mathrm{~m}^{2}$, potential inaccuracy of MDRD at higher levels of GFR was not a major consideration. CKD stage was defined from eGFR according to Kidney Disease: Improving Global Outcomes (KDIGO) 2012 clinical practice guideline for the evaluation and management of chronic kidney disease. As data on urinalysis and cystatin $\mathrm{C}$ was not available for the purposes of this study, presence of CKD stage 3 was defined as eGFR $45-60 \mathrm{~mL} \min .1 .73 \mathrm{~m}^{2}$.

\section{CPET performance}

Investigators blinded to CKD stage carried out all exercise testing. Equipment was calibrated before each test using standard reference gases. Patients undertook CPET on an electronically controlled cycle ergometer (Zan, nSpire, Colorado, USA; Lode, Groningen, The Netherlands) to maximal tolerance, adhering to the timed protocol detailed in online supplementary figure S1. Continuous 12-lead ECG was recorded. Continuous breath-by-breath gas exchange analysis was performed, with on-line calculations of oxygen consumption $\left(\mathrm{VO}_{2}\right.$; $\mathrm{mL} / \mathrm{kg} / \mathrm{min}$ ), minute ventilation (VE), carbon dioxide production $\left(\mathrm{VCO}_{2}\right)$ and respiratory exchange ratio (RER; $\mathrm{VCO}_{2} / \mathrm{VO}_{2}$ ). Before each test, the pneumotachometer was referenced by calibrating the gas analysers with a certified air mixture, according to the manufacturer's specifications. All patients were instructed to continue cycling until symptom-limited fatigue. CPET data were analysed blinded to CKD status. Anaerobic threshold (AT) was determined by two independent assessors blinded to autonomic parameters, and according to published guidelines using the modified V-slope method, and confirmed by ventilatory equivalents for carbon dioxide $\left(\dot{\mathrm{V}}_{\mathrm{E}} / \dot{\mathrm{V}}_{\mathrm{CO}_{2}}\right)$ and oxygen $\left(\mathrm{V}_{\mathrm{E}} / \dot{\mathrm{V}}_{\mathrm{O}_{2}}\right) .{ }^{16}{ }^{17}$ Peak oxygen consumption $\left(\dot{\mathrm{V}}_{\mathrm{O}_{\text {peak }}}\right)$, oxygen pulse and $\dot{\mathrm{V}}_{\mathrm{E}} / \dot{\mathrm{V}}_{\mathrm{CO}_{2}}$ were also recorded. $\dot{\mathrm{V}}_{\mathrm{E}} / \dot{\mathrm{V}}_{\mathrm{CO}_{2}}$ is inversely related to cardiac output at peak exercise, ${ }^{18}$ and is a better predictor of outcome in heart failure. ${ }^{19}$ Predicted values, derived from population norms that take into account age, gender, weight and height, were calculated for $\dot{\mathrm{V}}_{\mathrm{O}_{2 \text { peak }}}{ }^{20}$ and oxygen pulse, ${ }^{21}$ a robust measure of left ventricular stroke volume. ${ }^{22}{ }^{23}$ Per cent predicted peak $\dot{\mathrm{V}} \mathrm{O}_{2}$ values (derived from the Wasserman/Hansen equations) are a superior measure of these variables, at least in patients with established heart failure. ${ }^{24}$

\section{Assessment of exercise-evoked ischaemia}

Continuous 12-lead ECG recordings were made throughout the CPET period to enable the detection of ischaemia and/or development of dysrhythmias. ST-segment depression was quantified in lead II, which is superior for detection of atrial dysrhythmias, and more easily obtained with conventional monitors. ${ }^{25}$ Lead II ST changes were defined as abnormal when ST depression of $0.1 \mathrm{mV}(1 \mathrm{~mm})$ or more occurred, in accordance with current American College of Cardiology guidelines, ${ }^{26}$ and consistent with previous studies identifying that ST-segment depression to levels $\geq 1 \mathrm{~mm}$ independently predict future cardiac events in asymptomatic populations. ${ }^{27}$

\section{Autonomic assessment}

Autonomic data were analysed offline, blinded to CKD status and postoperative outcomes. Exercise-evoked sympathetic and parasympathetic autonomic responses were assessed following the CPET protocol as described above. Patients undertook 3 min of unloaded pedalling prior to the initiation of ramped exercise. Heart rate readings were obtained via ECG with the patient sitting on the cycle ergometer. All patients acclimatised for $3 \mathrm{~min}$ on the ergometer prior to start exercise. Non-invasive blood pressure was measured at the start (zero workload) and at the end of CPET. Sympathetic dysfunction was defined as the early acceleration of heart rate prior to exercise (AHRR), that is, the difference between the heart rate at rest and the heart rate measured just before starting loaded pedalling during the exercise test protocol (ie, after 3 min of unloaded exercise). Parasympathetic dysfunction was defined from heart rate recovery at $1 \mathrm{~min}$ following the cessation of exercise. We defined an abnormal heart rate recovery as a failure to decrease heart rate by $\geq 12 \mathrm{bpm} 1 \mathrm{~min}$ after the end of exercise, based on extensive previous data showing an association between heart rate recovery and an increased risk of cardiovascular and all-cause cardiovascular death. ${ }^{28} 29$

\section{Statistics}

The primary outcome was $\dot{\mathrm{V}}_{\mathrm{O}_{\text {ppeak }}}$. Secondary outcomes were AT, $\dot{\mathrm{V}}_{\mathrm{E}} / \dot{\mathrm{V}}_{\mathrm{CO}_{2}}$, oxygen pulse, anticipatory heart rate and heart rate recovery. Baseline characteristics of participants were compared according to presence of CKD stage 3 using general linear models of analysis of variance (quantitative variables), controlling for age as a covariate where CPET variables are not referenced to population norms. Non-parametric data were analysed with the Kruskal-Wallis test. Categorical variables were analysed using Fisher's exact test. Multivariate analysis to establish clinical predictors of peak $\mathrm{VO}_{2}$ took into 
account age (continuous variable) and the following categorical variables: gender, malignancy, hypertension, cardiac failure, diabetes mellitus and CKD stage 3. All reported $p$ values are two sided, with significance set at $\mathrm{p} \leq 0.05$. Statistical analyses were performed using NCSS 8 (Kaysville, Utah, USA).

\section{Sample size calculation}

On the basis of previous cardiac structural studies, a prevalence of $48 \%$ left ventricular hypertrophy (LVH) occurs in CKD stage 3, versus $32 \%$ in patients with normal renal function. Approximately $10 \%$ of UK patients have CKD in the general population. We estimated that LVH would mirror the degree of impaired exercise performance; therefore, to detect a similar difference in lower-than-normal predicted $\dot{\mathrm{V}}_{\mathrm{O}_{2 \text { peak }}}$, a sample size $\geq 853$ patients was required ( $\alpha$ of 0.05 ; power of $80 \%)$. A $10 \%$ dropout rate was anticipated for patients in whom AT was difficult to assess, or who failed to complete CPET for medical reasons (arrhythmia, (pre-) syncope).

\section{RESULTS}

\section{Clinical characteristics}

We analysed CPET data for 933 patients (figure 1). CKD stage 3 was present in 93/933 (9.97\%) patients. Demographics for patients are shown in table 1, stratified by the presence/absence of CKD stage 3. Patients were well matched for biochemical biomarkers

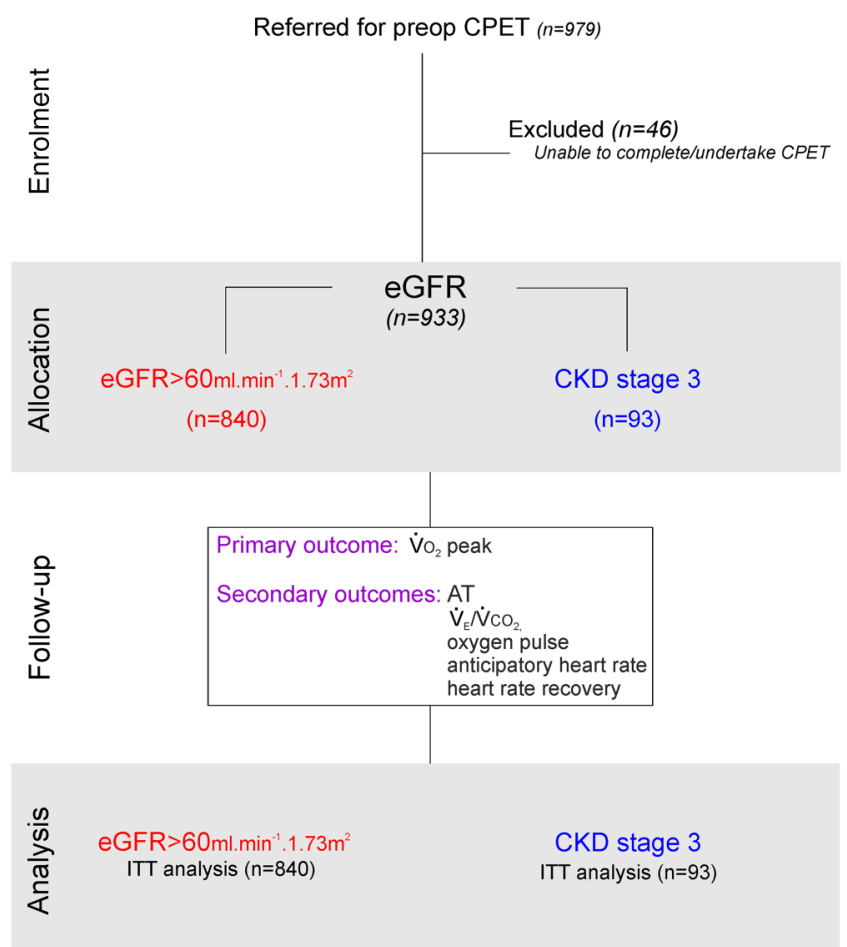

Figure 1 Study analysis plan. CKD, chronic kidney disease; CPET, cardiopulmonary exercise testing; eGFR, estimated glomerular filtration rate; ITT, intention to treat. synonymous with advanced CKD and increased risk of cardiovascular disease, ${ }^{30}$ namely, similar haemoglobin and albumin levels. Diabetes mellitus (RR 2.49 (95\% CI 1.59 to 3.89 ); $\mathrm{p}<0.001$ ), and hypertension (RR 3.20 (95\% CI 2.04 to 5.03$) ; \mathrm{p}<0.001)$ ) were more prevalent in CKD stage 3. However, clinically diagnosed/treated cardiac failure (RR 0.83 (95\% CI 0.30 to 2.24); $\mathrm{p}=0.70$ ) and ischaemic heart disease (RR 1.40 (95\% CI 0.97 to 2.02); $\mathrm{p}=0.09$ ) were not more prevalent in CKD3.

\section{Functional cardiovascular reserve in CKD3 versus normal renal function}

Cardiopulmonary parameters measured during exercise are summarised in table 2. Consistent with their clinical cardiovascular phenotype, neither left ventricular stroke volume (predicted oxygen pulse; mean difference: $6 \%$ ( -2 to 13$) ; \mathrm{p}=0.33$ ) nor exercise-evoked ischaemia differed between patients with CKD3 and normal renal function. These data were consistent with a low prevalence of clinically diagnosed heart failure $(<5 \%$ in both groups).

However, despite a similar prevalence of overt cardiovascular disease, CPET revealed that patients with CKD stage 3 had lower predicted values for $\mathrm{AT}$ and $\mathrm{VO}_{2 \text { peak }}$, which control for age, gender, weight and height. Predicted $\mathrm{VO}_{\text {2peak }}$ (mean difference: $6 \%$ (95\% CI $1 \%$ to $11 \%) ; \mathrm{p}=0.02$ ) and lower AT (mean difference: $1.1 \mathrm{~mL} /$ $\mathrm{min} / \mathrm{kg}(95 \%$ CI 0.4 to 1.7$) ; \mathrm{p}=0.002)$ were lower in patients with CKD stage 3. Ventilatory equivalents for carbon dioxide $\left(\dot{\mathrm{V}}_{\mathrm{E}} / \dot{\mathrm{V}}_{\mathrm{CO}_{2}}\right)$, a powerful predictor of prognosis in clinically diagnosed heart failure, was substantially higher in patients with CKD stage 3 (mean difference: 3.5 (95\% CI 2.4 to 4.6$)$; $\mathrm{p}<0.001)$. Taken together, these data suggest that patients with CKD stage 3 have occult cardiovascular dysfunction that is not revealed by clinical history or routine tests.

\section{Autonomic characteristics of CKD3}

Online supplementary figure S1 summarises measures of heart rate dynamics made during CPET over time in patients with normal renal function and those with CKD stage 3. Similar resting heart rates were evident in patients with normal renal function and those with CKD stage 3. Table 3 summarises individualised changes in absolute heart rate indicative of sympathetic (peak and anticipatory) and parasympathetic (recovery) autonomic function. Patients with CKD stage 3 had lower peak heart rates attained during exercise (mean difference: $9 \mathrm{bpm}(95 \%$ CI 3 to 14$) ; \mathrm{p}=0.002)$ ) and impaired heart rate recovery (HRR; online supplementary figure S1) from peak exercise heart rate (mean difference: $4 \mathrm{bpm}$ (95\% CI 1 to 7$) ; \mathrm{p}=0.003)$ ). Controlling for age, the smaller increases in peak heart rate from baseline (rise; figure 1), and lower heart rate recovery observed in patients with CKD stage 3 remained significant. Heart rate recovery in patients with CKD stage 3 was more likely to fall below $12 \mathrm{bpm}$ (relative risk: 1.51 (95\% CI 1.17 to 1.96$) ; \mathrm{p}<0.001)$, a threshold value which has 
Table 1 Preoperative demographics/comorbidities for study population

\begin{tabular}{|c|c|c|c|}
\hline & eGFR $>60$ & CKD stage 3 & p Value \\
\hline $\mathrm{n}$ & 840 & 93 & \\
\hline Age (years) & 61 (59 to 61$)$ & 71 (69 to 73 ) & $<0.001$ \\
\hline Gender ( $\mathrm{n} ; \%$ male $)$ & $546(65)$ & $69(74)$ & 0.08 \\
\hline BMI $\left(\mathrm{kg} / \mathrm{m}^{2}\right)$ & 26.7 (26.3 to 27.1 ) & 27.4 (26.3 to 28.5 ) & 0.24 \\
\hline Cancer (n (\%)) & $360(43)$ & $34(37)$ & 0.34 \\
\hline Ischaemic heart disease (n (\%)) & $156(19)$ & $24(26)$ & 0.08 \\
\hline Hypertension (n (\%)) & $252(30)$ & $51(55)$ & $<0.001$ \\
\hline Heart failure (n (\%)) & $44(5)$ & $4(4)$ & 0.44 \\
\hline CVA/TIA (n (\%)) & $20(2)$ & $4(4)$ & 0.31 \\
\hline Diabetes mellitus ( $\mathrm{n}(\%)$ ) & 71 (9) & $20(22)$ & $<0.001$ \\
\hline Haemoglobin $(\mathrm{g} / \mathrm{dL})$ & $13.3(13.0$ to 13.5$)$ & $12.9(12.5$ to 13.3$)$ & 0.39 \\
\hline Albumin ( $g / L)$ & $44 \pm 4$ & $44 \pm 4$ & 0.81 \\
\hline Lung disease/COPD (n (\%)) & $98(13)$ & $15(16)$ & 0.49 \\
\hline Smoker (ex/present; n (\%) & 291 (35) & $36(39)$ & 0.55 \\
\hline$\beta$-blocker (n (\%)) & $85(10)$ & $13(14)$ & 0.20 \\
\hline Nitrate $(\mathrm{n}(\%))$ & $24(3)$ & $5(5)$ & 0.16 \\
\hline ACE inhibitor (n (\%)) & $119(14)$ & $26(28)$ & $<0.001$ \\
\hline Statin $(n(\%))$ & $184(22)$ & $34(37)$ & $<0.001$ \\
\hline
\end{tabular}

Data are shown as $\mathrm{n}(\%)$ or mean $(95 \% \mathrm{Cls})$.

BMI, body mass index; CKD, chronic kidney disease; COPD, chronic obstructive pulmonary disease; CVA, cerebral vascular accident; eGFR, estimated glomerular filtration rate; TIA, transient ischemic attack.

been shown to be an independent predictor of all-cause and cardiovascular-related mortality. ${ }^{28}{ }^{29}$ Anticipatory heart rate (AHRR; online supplementary figure S1) during unloaded pedalling, a measure of sympathetic dysfunction, ${ }^{31}$ was similar between groups.

\section{Functional cardiovascular reserve in CKD3 with/without essential hypertension}

End-stage CKD is associated with lower exercise capacity compared to patients with hypertension. ${ }^{6}$ On subgroup analysis, we found that patients with CKD stage 3 without hypertension had lower exercise capacity compared to non-hypertensive patients with normal renal function $(n=516$; table 4). Similar results were obtained for patients with CKD stage 3 without diabetes mellitus (see online supplementary data). Multivariate analysis

\begin{tabular}{lccc} 
Table 2 & CPET performance for study population \\
\hline & eGFR $>60$ & CKD stage 3 & p Value \\
\hline $\mathrm{VO}_{2 \text { peak }}(\mathrm{mL} / \mathrm{min} \mathrm{kg})$ & $17.7 \pm 5.6$ & $14.9 \pm 4.1$ & $<0.001$ \\
$\mathrm{VO}_{2 \text { peak }}(\%$ predicted) & $76 \pm 25$ & $69 \pm 18$ & 0.02 \\
$\mathrm{VO}_{2} \mathrm{AT}(\mathrm{mL} / \mathrm{min} \mathrm{kg})$ & $11.4 \pm 2.2$ & $10.4 \pm 2.2$ & $<0.001$ \\
$\mathrm{VE}-\mathrm{VCO}_{2}$ slope & $30.3 \pm 5.9$ & $33.8 \pm 5.9$ & $<0.001$ \\
Oxygen pulse & $10.2 \pm 4.2$ & $9.1 \pm 2.6$ & 0.35 \\
(mL/min $\mathrm{O}_{2}$ ) & & & \\
Oxygen pulse & $90.6 \pm 26$ & $85.1 \pm 21$ & 0.35 \\
(\% predicted) & & & \\
\hline
\end{tabular}

Data are shown as mean (SD). All \% predicted values control for age, gender, weight.

AT, Anaerobic threshold; CKD, chronic kidney disease; CPET, cardiopulmonary exercise testing; eGFR, estimated glomerular filtration rate; $\mathrm{VCO}_{2}$, carbon dioxide production; VE, ventilation; $\mathrm{VO}_{2 \text { peak }}$, peak oxygen consumption. identified CKD stage 3 and pre-existing cardiac failure as independent predictors of $\mathrm{VO}_{2 \text { peak }}$ (table 5), taking into account coexistence of diabetes mellitus and hypertension.

\section{DISCUSSION}

These data show that CPET reveals subclinical differences in cardiopulmonary physiology in patients with CKD stage 3. Patients with CKD stage 3 did not have overt clinical histories or evidence for inferior cardiovascular performance. These data are the first to demonstrate that early CKD is associated with impaired cardiopulmonary reserve in the absence of known systolic heart failure. Importantly, our analyses used population norms for cardiopulmonary exercise performance controlling for age and gender, in contrast with a comparable study exploring the relationship between CKD and exercise capacity in patients with known systolic heart failure ${ }^{10}$ Furthermore, these cardiopulmonary differences appear to precede established biomarkers for

Table 3 Dynamic heart rate changes during CPET eGFR $>60$ CKD stage $3 \mathrm{p}$ Value

\begin{tabular}{lrcr}
\hline Baseline heart rate & $84 \pm 15$ & $85 \pm 16$ & 0.49 \\
Anticipatory heart rate & $11 \pm 10$ & $12 \pm 9$ & 0.15 \\
Peak heart rate & $138 \pm 24$ & $130 \pm 20$ & 0.002 \\
Heart rate rise & $57 \pm 24$ & $45 \pm 21$ & $<0.001$ \\
Heart rate recovery & $19 \pm 13$ & $15 \pm 8$ & 0.001
\end{tabular}
at $1 \mathrm{~min}$

Data are shown as mean (SD)

CKD, chronic kidney disease; CPET, cardiopulmonary exercise testing; eGFR, estimated glomerular filtration rate. 
Table 4 CPET characteristics of non-hypertensive patients with/without CKD stage 3

eGFR $>60$ CKD stage 3 p Value

\begin{tabular}{lrcc}
\hline $\mathrm{VO}_{\text {2peak }}(\mathrm{mL} / \mathrm{min} \mathrm{kg})$ & $18.7 \pm 5.9$ & $15.2 \pm 4.4$ & 0.001 \\
$\mathrm{VO}_{2 \text { peak }}(\%$ predicted) & $75 \pm 24$ & $66 \pm 16$ & 0.02 \\
$\mathrm{VO}_{2} \mathrm{AT}(\mathrm{mL} / \mathrm{min} \mathrm{kg})$ & $11.7 \pm 3.1$ & $10.6 \pm 2.7$ & 0.01 \\
$\mathrm{VE}^{\mathrm{V} \mathrm{VCO}_{2} \text { slope }}$ & $29.7 \pm 4.9$ & $34.8 \pm 6.6$ & $<0.001$ \\
$\begin{array}{l}\text { Oxygen pulse } \\
\left(\mathrm{mL} / \mathrm{min} \mathrm{O}_{2}\right)\end{array}$ & $10.3 \pm 4.6$ & $8.9 \pm 3.1$ & 0.21 \\
$\begin{array}{l}\text { Oxygen pulse } \\
\text { (\% predicted) }\end{array}$ & $90 \pm 28$ & $82 \pm 23$ & 0.21 \\
$\begin{array}{l}\text { Peak heart rate (bpm) } \\
\text { Heart rate }\end{array}$ & $142 \pm 24$ & $131 \pm 19$ & 0.02 \\
recovery (bpm) & $20 \pm 13$ & $16 \pm 9$ & 0.05 \\
\hline
\end{tabular}

Data are shown mean (SD). All \% predicted values control for age, gender, weight.

AT, Anaerobic threshold; CKD, chronic kidney disease; CPET, cardiopulmonary exercise testing; eGFR, estimated glomerular filtration rate; $\mathrm{VCO}_{2}$, carbon dioxide production; VE, ventilation; $\mathrm{VO}_{2 \text { peak }}$, peak oxygen consumption.

cardiovascular disease in more advanced CKD, including albumin and haemoglobin. ${ }^{30}$ These findings suggest that impaired exercise performance begins at a much earlier stage of CKD in keeping with cardiac structural insights provided by echocardiography. ${ }^{13}$ Although approximately half the patients studied had malignancy, this was not associated with the inferior exercise performance evident in CKD stage 3.

CKD increases the risk of acquiring ${ }^{32} 33$ and accelerates the progression of chronic heart failure. ${ }^{34-37}$ The pathophysiology of cardiorenal syndromes is a complex bidirectional process. Our findings provide functional physiological data using a gold standard assessment of cardiopulmonary functions that are consistent with previous morphological studies detailing subclinical cardiac abnormalities in CKD. ${ }^{13}$ Left ventricular hypertrophy is more common in patients with CKD, which has previously been thought to precede functional impairment. ${ }^{35}$ These structural differences appear to be driven by progressive CKD, in part, accounted for by the increased

\begin{tabular}{|c|c|c|c|c|}
\hline \multirow[b]{2}{*}{$\begin{array}{l}\text { Independent } \\
\text { variable }\end{array}$} & \multirow[b]{2}{*}{$\begin{array}{l}\text { Regression } \\
\text { coefficient }\end{array}$} & \multicolumn{2}{|l|}{$95 \% \mathrm{CL}$} & \multirow[b]{2}{*}{$\begin{array}{l}p \\
\text { Value }\end{array}$} \\
\hline & & Lower & Upper & \\
\hline Intercept & 74.9678 & 72.4250 & 77.5106 & 0.01 \\
\hline Cancer & 1.2933 & -2.0560 & 4.6426 & 0.45 \\
\hline Cardiac failure & -20.1919 & -35.3029 & -5.0810 & 0.01 \\
\hline CKD stage 3 & -6.2930 & -11.9994 & -0.5866 & 0.03 \\
\hline $\begin{array}{l}\text { Diabetes } \\
\text { mellitus }\end{array}$ & -4.9127 & -10.5744 & 0.7491 & 0.09 \\
\hline Hypertension & 0.7507 & -2.9437 & 4.4451 & 0.69 \\
\hline
\end{tabular}

prevalence of diabetes mellitus and hypertension in CKD. Our data are consistent with recent epidemiological work showing that CKD confers greater risk of cardiovascular disease than diabetes, ${ }^{38}$ since patients with diabetes in this cohort free of CKD had superior cardiopulmonary exercise performance. Our data suggest that CKD has a further negative impact on poor cardiovascular function.

Because the prevalence of hypertension in patients with CKD is very common, we also compared patients free of, or with established, hypertension. In this large subset, we confirmed the overall findings that patients with CKD stage 3 without hypertension had lower exercise capacity compared to non-hypertensive controls. Furthermore, analysis of exercise-evoked changes in heart rate revealed distinct autonomic profiles in CKD stage 3 . The finding that peak heart rate was impaired mirrors a similar observation in patients with end-stage CKD, in whom a blunted chronotropic response was an independent predictor of peak $\mathrm{VO}_{2}{ }^{6}$ Furthermore, impaired parasympathetic dysfunction-as suggested by slower heart rate recovery in patients CKD-has emerged as an important contributor to myocardial injury, ${ }^{39}$ systemic inflammation ${ }^{40}$ and progression of CKD. ${ }^{41}{ }^{42}$ Interestingly, patients with CKD stage 3 were more likely to have an HRR value previously shown to predict mortality and development of significant cardiovascular morbidity/mortality. ${ }^{28} 29$

Significant limitations include the observational crosssectional nature of the study which prevents more robust conclusions regarding causality, particularly given limited information on the duration of key comorbid conditions including hypertension and diabetes. Echocardiographic correlates of exercise performance may provide further structural and functional insights, although static measures would not provide the depth of physiological insight afforded by CPET. Strengths of these data are reflected by the large data set, blinded study design, appropriately powered analysis plan, and established biological plausibility for the cardiovascular phenotypes described.

There are several clinical implications generated by these data. The presence of CKD stage 3 should alert clinicians to early CKD being a surrogate marker for poorer cardiac output under stress. CKD stage 3 is associated with poorer outcomes following major noncardiac and cardiac surgery. ${ }^{43}$ Thus, acute blood loss, hypotension ${ }^{44}$ and/or hypovolemia in patients with CKD stage 3 may confer additional increased risk of pan-organ and further renal injury as a result of inferior cardiovascular performance. Further studies are required to determine whether the exercise phenotype maps to inferior cardiovascular performance in various clinical situations.

In summary, patients with CKD stage 3, but without clinical heart failure, have significantly worse cardiovascular function when challenged with standardised, protocolised physiological stress. In addition to the higher 
prevalence of diabetes mellitus and hypertension evident in patients with CKD stage 3, the subclinical dysfunctional cardiopulmonary phenotype described here provides additional pathophysiological mechanisms that may contribute to poorer clinical outcomes following acute cardiometabolic stress.

Contributors GLA contributed to study design and data analysis. JW, AN and JMO contributed to data collection and data analysis. RS and DSM contributed to CPET data collection and data analysis. GLA and JP wrote the manuscript.

Funding This project was funded in part by a British Journal of Anaesthesia/ Royal College of Anaesthetists John Snow Intercalated BSc award (AN); British Journal of Anaesthesia/Royal College of Anaesthetists Career Development Basic Science Fellowship award (GLA); National Institute for Health Research Central and East London Clinical Research Network. This work was undertaken at University College London Hospitals NHS Trust/ University College London who received a proportion of funding from the Department of Health UK NIHR Biomedical Research Centre funding scheme.

Competing interests None declared.

Ethics approval MREC: 12/LO/0453.

Provenance and peer review Not commissioned; externally peer reviewed.

Data sharing statement Data is available on request to bone fide researchers from the corresponding author.

Open Access This is an Open Access article distributed in accordance with the Creative Commons Attribution Non Commercial (CC BY-NC 4.0) license, which permits others to distribute, remix, adapt, build upon this work noncommercially, and license their derivative works on different terms, provided the original work is properly cited and the use is non-commercial. See: http:// creativecommons.org/licenses/by-nc/4.0/

\section{REFERENCES}

1. Hallan SI. Kidney function for the non-nephrologist: an emerging tool for predicting mortality risk. Kidney Int 2011;79:8-10.

2. de Jager DJ, Vervloet MG, Dekker FW. Noncardiovascular mortality in CKD: an epidemiological perspective. Nat Rev Nephrol 2014:10:208-14.

3. Muntner $P$, Judd SE, Gao L, et al. Cardiovascular risk factors in CKD associate with both ESRD and mortality. J Am Soc Nephrol 2013;24:1159-65.

4. Bello AK, Hemmelgarn B, Lloyd A, et al. Associations among estimated glomerular filtration rate, proteinuria, and adverse cardiovascular outcomes. Clin J Am Soc Nephrol 2011;6:1418-26.

5. Herzog CA, Asinger RW, Berger AK, et al. Cardiovascular disease in chronic kidney disease. A clinical update from Kidney Disease: Improving Global Outcomes (KDIGO). Kidney Int 2011;80:572-86.

6. Ting SM, Hamborg T, McGregor G, et al. Reduced Cardiovascular Reserve in Chronic Kidney Failure: A Matched Cohort Study. Am J Kidney Dis 2015;66:274-84

7. Sietsema KE, Amato A, Adler SG, et al. Exercise capacity as a predictor of survival among ambulatory patients with end-stage renal disease. Kidney Int 2004;65:719-24.

8. Sezer S, Elsurer R, Ulubay G, et al. Factors associated with peak oxygen uptake in hemodialysis patients awaiting renal transplantation. Transplant Proc 2007;39:879-82.

9. Ting SM, Iqbal $\mathrm{H}$, Kanji $\mathrm{H}$, et al. Functional cardiovascular reserve predicts survival pre-kidney and post-kidney transplantation. J Am Soc Nephrol 2014;25:187-95.

10. McCullough PA, Franklin BA, Leifer E, et al. Impact of reduced kidney function on cardiopulmonary fitness in patients with systolic heart failure. Am J Nephrol 2010;32:226-33.

11. Poulikakos D, Banerjee D, Malik M. Repolarisation descriptors and heart rate variability in hemodialysed patients. Physiol Res 2015;64:487-93.

12. Poulikakos D, Banerjee D, Malik M. Risk of sudden cardiac death in chronic kidney disease. J Cardiovasc Electrophysiol 2014;25:222-31.

13. Park M, Hsu CY, Li Y, et al. Associations between kidney function and subclinical cardiac abnormalities in CKD. J Am Soc Nephrol 2012;23:1725-34.
14. American Thoracic Society; American College of Chest Physicians ATS/ACCP Statement on cardiopulmonary exercise testing. Am J Respir Crit Care Med 2003;167:211-77.

15. Levey AS, Bosch JP, Lewis JB, et al. A more accurate method to estimate glomerular filtration rate from serum creatinine: a new prediction equation. Modification of Diet in Renal Disease Study Group. AnnInternMed 1999;130:461-70.

16. Beaver WL, Wasserman K, Whipp BJ. A new method for detecting anaerobic threshold by gas exchange. J Appl Physiol 1986;60:2020-7.

17. Pina IL, Balady GJ, Hanson P, et al. Guidelines for clinical exercise testing laboratories. A statement for healthcare professionals from the Committee on Exercise and Cardiac Rehabilitation, American Heart Association. Circulation 1995;91:912-21.

18. Reindl I, Wernecke KD, Opitz C, et al. Impaired ventilatory efficiency in chronic heart failure: possible role of pulmonary vasoconstriction. Am Heart $J$ 1998;136:778-85.

19. Chua TP, Ponikowski P, Harrington D, et al. Clinical correlates and prognostic significance of the ventilatory response to exercise in chronic heart failure. J Am Coll Cardiol 1997;29:1585-90.

20. Hansen JE, Sue DY, Wasserman K. Predicted values for clinical exercise testing. Am Rev Respir Dis 1984;129:S49-55.

21. Uth N, Sørensen $\mathrm{H}$, Overgaard K, et al. Estimation of VO2max from the ratio between HRmax and HRrest-the Heart Rate Ratio Method. Eur J Appl Physiol 2004;91:111-15.

22. Whipp BJ, Higgenbotham MB, Cobb FC. Estimating exercise stroke volume from asymptotic oxygen pulse in humans. J Appl Physiol 1996;81:2674-9.

23. Oelberg DA, Kacmarek RM, Pappagianopoulos PP, et al. Ventilatory and cardiovascular responses to inspired $\mathrm{He}-\mathrm{O} 2$ during exercise in chronic obstructive pulmonary disease. Am J Respir Crit Care Med 1998;158:1876-82.

24. Arena R, Myers J, Abella J, et al. Determining the preferred percent-predicted equation for peak oxygen consumption in patients with heart failure. Circ Heart Fail 2009;2:113-20.

25. London MJ, Hollenberg M, Wong MG, et al. Intraoperative myocardial ischemia: localization by continuous 12-lead electrocardiography. Anesthesiology 1988;69:232-41.

26. Fihn SD, Gardin JM, Abrams J, et al. 2012 ACCF/AHA/ACP/AATS/ PCNA/SCAI/STS Guideline for the diagnosis and management of patients with stable ischemic heart disease: a report of the American College of Cardiology Foundation/American Heart Association Task Force on Practice Guidelines, and the American College of Physicians, American Association for Thoracic Surgery, Preventive Cardiovascular Nurses Association, Society for Cardiovascular Angiography and Interventions, and Society of Thoracic Surgeons. J Am Coll Cardiol 2012;60:e44-e164.

27. Rywik TM, O'Connor FC, Gittings NS, et al. Role of nondiagnostic exercise-induced ST-segment abnormalities in predicting future coronary events in asymptomatic volunteers. Circulation 2002;106:2787-92.

28. Cole CR, Blackstone EH, Pashkow FJ, et al. Heart-rate recovery immediately after exercise as a predictor of mortality. N Engl J Med 1999;341:1351-7.

29. Jouven X, Empana JP, Schwartz PJ, et al. Heart-rate profile during exercise as a predictor of sudden death. N Engl J Med 2005;352:1951-8

30. Joshi S, Viljoen A. Renal biomarkers for the prediction of cardiovascular disease. Curr Opin Cardiol 2015;30:454-60.

31. Whittle J, Nelson A, Otto JM, et al. Sympathetic autonomic dysfunction and impaired cardiovascular performance in higher risk surgical patients: implications for perioperative sympatholysis. Open Heart 2015;2:e000268.

32. Bibbins-Domingo K, Lin F, Vittinghoff $\mathrm{E}$, et al. Predictors of heart failure among women with coronary disease. Circulation 2004:110:1424-30.

33. Chae CU, Albert CM, Glynn RJ, et al. Mild renal insufficiency and risk of congestive heart failure in men and women $>$ or $=70$ years of age. Am J Cardiol 2003:92:682-6.

34. Bongartz LG, Cramer MJ, Doevendans PA, et al. The severe cardiorenal syndrome: 'Guyton revisited'. Eur Heart J 2005;26: 11-7.

35. Paoletti E, Bellino D, Cassottana P, et al. Left ventricular hypertrophy in nondiabetic predialysis CKD. Am J Kidney Dis 2005:46:320-7.

36. Levin A, Thompson CR, Ethier J, et al. Left ventricular mass index increase in early renal disease: impact of decline in hemoglobin. Am J Kidney Dis 1999;34:125-34.

37. Bibbins-Domingo K, Lin F, Vittinghoff $\mathrm{E}$, et al. Renal insufficiency as an independent predictor of mortality among women with heart failure. J Am Coll Cardiol 2004;44:1593-600. 
38. Tonelli M, Muntner P, Lloyd A, et al. Risk of coronary events in people with chronic kidney disease compared with those with diabetes: a population-level cohort study. Lancet 2012;380: 807-14.

39. Mastitskaya S, Marina N, Gourine A, et al. Cardioprotection evoked by remote ischaemic preconditioning is critically dependent on the activity of vagal pre-ganglionic neurones. Cardiovasc Res 2012;95:487-94

40. Andersson U, Tracey KJ. Reflex principles of immunological homeostasis. Annu Rev Immunol 2012;30:313-35.
41. Brotman DJ, Bash LD, Qayyum R, et al. Heart rate variability predicts ESRD and CKD-related hospitalization. J Am Soc Nephrol 2010;21:1560-70.

42. Yeboah MM, Xue X, Duan B, et al. Cholinergic agonists attenuate renal ischemia-reperfusion injury in rats. Kidney Int 2008;74:62-9.

43. Ackland GL, Moran N, Cone S, et al. Chronic kidney disease and postoperative morbidity after elective orthopedic surgery. Anesth Analg 2011;112:1375-81.

44. Asfar P, Meziani F, Hamel JF, et al. High versus low blood-pressure target in patients with septic shock. N Engl J Med 2014;370:1583-93. 